\title{
Possible ion-acoustic soliton formation in the ionospheric perturbations observed on DEMETER before the 2007 Pu'er earthquake*
}

\author{
U. A. Mofiz ${ }^{1, \uparrow}$ and R. Battiston ${ }^{2}$ \\ ${ }^{1}$ Department of Mathematics and Natural Sciences, BRAC University, 66 Mohakhali, Dhaka 1212, Bangladesh \\ ${ }^{2}$ Istituto Nazionale di Fisica Nucleare, Via A. Pascoli, Perugia 06123, Italy
}

\begin{abstract}
The data of ionospheric perturbations observed on DEMETER before the $2007 \mathrm{Pu}$ 'er earthquake are analyzed. The three-component plasma (ions, electrons and heavy ions) is studied in the fluid concept. The linear dispersion relation for ion-acoustic wave is found in the presence of heavy ions. The nonlinear dynamics is studied for arbitrary amplitude of the wave. The Sagdeev potential is calculated, which shows that solitary structure exists for Mach number within a range defined by the presence of heavy ions. The developed ion-acoustic solitons may be used as precursor for earthquake prediction.
\end{abstract}

Key words: earthquake; ionosphere; plasma; dispersion; ion-acoustic soliton CLC number: P352.3 Document code: A

\section{Introduction}

Detection of electromagnetic emission transmitted from earthquake regions (DEMETER) is a micro-satellite built by the Centre National d'Etudies Spatiales (CNES) of French launched on 29 June 2004 on a nearly circular $\sim 710 \mathrm{~km}$ altitude quasi-heliosynchron at $98^{\circ}$ inclination. Measurements of the electric and magnetic field components of the electromagnetic waves are performed by the instrument champ electrique (ICE) (Berthelier et al, 2006) and by the instrument magnetic search coil (IMSC) (Parrot et al, 2006), respectively.

A destructive shock with magnitude of 6.3 occurred on 2 June 2007 at 21:34:56 (UTC) in Pu'er region $\left(23.0^{\circ} \mathrm{N}, 101.1^{\circ} \mathrm{E}\right)$, Yunnan province, China. The data from DEMETER satellite during the period from 23 May to 2 June, i.e., ten days before the earthquake and one day just on the day of earthquake occurrence, were analyzed (Zhu et al, 2008). Among the 284 orbits of DEMETER during the period, 29 orbits with the trace passing through the region within $1888 \mathrm{~km}$ from the epicenter were selected for the study. Seven anomalous

\footnotetext{
* Received 4 September 2008; accepted in revised form 12 May 2009; published 10 June 2009.

^. Corresponding author. e-mail: mofiz@bracu.ac.bd
}

events were found on the data set of the seven orbits among the 29 ones. There existed synchronous perturbations on the variations of the spectrogram of the electric field and the variations of the density and temperature of the ions and electron, in contrast with the variations of its surrounding area. Electrostatic turbulences were also recorded with the synchronous perturbations of the electron density and ion density in plasma in the region near the epicenter for the five events, which seems to suggest that there be some physical relation between these events and the preparation processes of the Pu'er earthquake.

In this paper, we will make further research on the density perturbations and electrostatic field turbulence by analyzing the three component plasma, i.e., ions, electrons and heavy ions, in the low temperature hydrodynamical fluid plasma concept. The linear dispersion relation of ion acoustic wave in the presence of heavy ion is derived. It is found that the phase velocity of the ion-acoustic wave decreases with heavy ion density increasing. For arbitrary amplitude of the perturbations, the nonlinear dynamics of the perturbed plasma is studied. The Sagdeev potential is calculated, which shows that ion-acoustic solitons are formed within a range of Mach number defined by the presence of heavy ions. We predict that the detection of the ion-acoustic solitons 
may be a precursor for earthquake prediction.

\section{Linear dynamics of ion-acoustic waves}

Consider a collisionless and unmagnetized three component plasma in the perturbed ionosphere due to seismic emission. The components are electrons (e), singly charged ions (i) and heavy ions (hi). The dynamics of the multi-component plasma is governed by the following fluid equations:

$$
m_{j} n_{j}\left[\frac{\partial \boldsymbol{V}_{j}}{\partial t}+\left(\boldsymbol{V}_{j} \cdot \nabla\right) \boldsymbol{V}_{j}\right]=-\nabla P_{j}+q_{j} n_{j} \boldsymbol{E},
$$

where, $j=\mathrm{i}$, e, hi; $P_{j}$ is the pressure, $q_{j}$ is the charge, $m_{j}$ is the mass and $n_{j}$ is the density of each species. $\boldsymbol{E}$ is the electrostatic field developed to balance the quasineutrality of the plasma.

For a quasi-neutral plasma, we have $n_{\mathrm{i}}+n_{\mathrm{hi}}=n_{\mathrm{e}} \equiv n$, which is maintained when $\boldsymbol{V}_{\mathrm{i}}=\boldsymbol{V}_{\mathrm{hi}}=\boldsymbol{V}_{\mathrm{e}} \equiv \boldsymbol{V}$ and $q_{\mathrm{i}}=q_{\mathrm{hi}}=-q_{\mathrm{e}}$. We consider the departure from charge neutrality is very small, then from momentum balance equation (1), we get

$$
\begin{aligned}
-\frac{1}{m_{\mathrm{i}}} \nabla P_{\mathrm{i}} & \frac{e}{m_{\mathrm{i}}} n_{\mathrm{i}} \boldsymbol{E}-\frac{1}{m_{\mathrm{hi}}} \nabla P_{\mathrm{hi}}+\frac{e}{m_{\mathrm{hi}}} n_{\mathrm{hi}} \boldsymbol{E}= \\
& -\frac{1}{m_{\mathrm{e}}} \nabla P_{\mathrm{e}}-\frac{e}{m_{\mathrm{e}}} n_{\mathrm{e}} \boldsymbol{E},
\end{aligned}
$$

which can be solved to get the electric field

$$
\boldsymbol{E}=\frac{\nabla\left(P_{\mathrm{i}}+\frac{m_{\mathrm{i}}}{m_{\mathrm{hi}}} P_{\mathrm{hi}}\right)-\frac{m_{\mathrm{i}}}{m_{\mathrm{e}}} \nabla P_{\mathrm{e}}}{e\left[\left(1+\frac{m_{\mathrm{i}}}{m_{\mathrm{e}}}\right) n-\left(1-\frac{m_{\mathrm{i}}}{m_{\mathrm{hi}}}\right) n_{\mathrm{hi}}\right]} .
$$

The motion equation of ions is

$$
m_{\mathrm{i}} n_{\mathrm{i}}\left[\frac{\partial \boldsymbol{V}_{\mathrm{i}}}{\partial t}+\left(\boldsymbol{V}_{\mathrm{i}} \cdot \nabla\right) \boldsymbol{V}_{\mathrm{i}}\right]=-\nabla P_{\mathrm{i}}+e n_{\mathrm{i}} \boldsymbol{E},
$$

which, with the substitutions $n_{\mathrm{i}}=n-n_{\text {hi }}$ and $\boldsymbol{V}_{\mathrm{i}} \sim \boldsymbol{V}$, gives

$$
\begin{gathered}
m_{\mathrm{i}}\left(n-n_{\mathrm{hi}}\right)\left[\frac{\partial \boldsymbol{V}}{\partial t}+(\boldsymbol{V} \cdot \nabla) \boldsymbol{V}\right]= \\
-\nabla P_{\mathrm{i}}+e\left(n-n_{\mathrm{hi}}\right) \boldsymbol{E} .
\end{gathered}
$$

Then substituting $\boldsymbol{E}$ from equation (3) into equation (5), we get

$$
m_{\mathrm{i}}\left(n-n_{\mathrm{hi}}\right)\left[\frac{\partial \boldsymbol{V}}{\partial t}+(\boldsymbol{V} \cdot \nabla) \boldsymbol{V}\right]=
$$

$$
\begin{aligned}
& -\left(1-\frac{n-n_{\mathrm{hi}}}{\left(1+\frac{m_{\mathrm{i}}}{m_{\mathrm{e}}}\right) n-\left(1-\frac{m_{\mathrm{i}}}{m_{\mathrm{hi}}}\right) n_{\mathrm{hi}}}\right) \nabla P_{\mathrm{i}}- \\
& \frac{m_{\mathrm{i}}}{m_{\mathrm{e}}} \frac{n-n_{\mathrm{hi}}}{\left(1+\frac{m_{\mathrm{i}}}{m_{\mathrm{e}}}\right) n-\left(1-\frac{m_{\mathrm{i}}}{m_{\mathrm{hi}}}\right) n_{\mathrm{hi}}} \nabla P_{\mathrm{e}}+ \\
& \frac{m_{\mathrm{i}}}{m_{\mathrm{hi}}} \frac{n-n_{\mathrm{hi}}}{\left(1+\frac{m_{\mathrm{i}}}{m_{\mathrm{e}}}\right) n-\left(1-\frac{m_{\mathrm{i}}}{m_{\mathrm{hi}}}\right) n_{\mathrm{hi}}} \nabla P_{\mathrm{hi}} .
\end{aligned}
$$

We consider the ion compression is adiabatic, electron compression is isothermal and the heavy ions are uncompressed. Then

$$
\begin{aligned}
& \nabla P_{\mathrm{i}}=\gamma T_{\mathrm{i}} \nabla n_{\mathrm{i}}= \\
& \gamma T_{\mathrm{i}} \nabla\left(n-n_{\mathrm{hi}}\right)=\gamma T_{\mathrm{i}} \nabla n \quad\left(n_{\mathrm{hi}}=n_{\text {hio }}\right), \\
& \nabla P_{\mathrm{e}}=T_{\mathrm{e}} \nabla n,
\end{aligned}
$$

and

$$
\nabla P_{\mathrm{hi}}=0,
$$

where $\gamma$ is the adiabatic constant and for one degree of freedom we consider $\gamma=3$.

With these assumptions, equation (6) reduces to

$$
\begin{gathered}
m_{\mathrm{i}}\left(n-n_{\mathrm{hi}}\right)\left[\frac{\partial \boldsymbol{V}}{\partial t}+(\boldsymbol{V} \cdot \nabla) \boldsymbol{V}\right]= \\
-\left(\lambda_{1} \gamma T_{\mathrm{i}}+\lambda_{2} T_{\mathrm{e}}\right) \nabla n,
\end{gathered}
$$

where

$$
\lambda_{1}=1-\frac{n-n_{\mathrm{hi} 0}}{\left(1+\frac{m_{\mathrm{i}}}{m_{\mathrm{e}}}\right) n-\left(1-\frac{m_{\mathrm{i}}}{m_{\mathrm{hi}}}\right) n_{\mathrm{hi} 0}}
$$

and

$$
\lambda_{2}=\frac{m_{\mathrm{i}}}{m_{\mathrm{e}}} \frac{n-n_{\mathrm{hi} 0}}{\left(1+\frac{m_{\mathrm{i}}}{m_{\mathrm{e}}}\right) n-\left(1-\frac{m_{\mathrm{i}}}{m_{\mathrm{hi}}}\right) n_{\text {hi } 0}} .
$$

The continuity equation of the fluid is

$$
\frac{\partial n}{\partial t}+\nabla \cdot\left(n-n_{\mathrm{hi} 0}\right) \boldsymbol{V}=0 .
$$

Equations (10) and (13) now can be used to determine the dispersion relation for the ion acoustic wave. We assume no average velocity of the medium, so that $\boldsymbol{V}$ is the velocity associated with the wave motion. Both the velocity and the density perturbation are considered to be small. If the undisturbed plasma density is $n_{0}$, then 
$n=n_{0}+\delta n$. We consider a plane wave propagation in the medium so that both $\delta n$ and $\boldsymbol{V}$ are proportional to $\exp [\mathrm{i}(\omega t-\boldsymbol{k} \cdot \boldsymbol{r})]$. Then we find the following dispersion relation:

$$
\omega^{2}=k^{2} C_{\mathrm{s}}^{2}\left(\lambda_{2}+\lambda_{1} \gamma \frac{T_{\mathrm{i}}}{T_{\mathrm{e}}}\right),
$$

where $C_{\mathrm{s}}=\left(T_{\mathrm{e}} / m_{\mathrm{i}}\right)^{1 / 2}$ is the sound speed. The phase velocity of the ion-acoustic wave is

$$
\frac{\omega}{k}=C_{\mathrm{s}} \sqrt{\lambda_{2}+\lambda_{1} \gamma \frac{T_{\mathrm{i}}}{T_{\mathrm{e}}}}
$$

or, we can write

$$
\frac{\omega}{k}=C_{\mathrm{s}}^{\prime} \quad \text { with } \quad C_{\mathrm{s}}^{\prime}=C_{\mathrm{s}} \lambda
$$

where

$$
\begin{aligned}
\lambda=\lambda\left(n_{0}, n_{\mathrm{hi} 0}, T_{\mathrm{i}}, T_{\mathrm{e}}\right)= & {\left[\frac{m_{\mathrm{i}}}{m_{\mathrm{e}}} \frac{1-\frac{n_{\mathrm{hi} 0}}{n_{0}}}{\left(1+\frac{m_{\mathrm{i}}}{m_{\mathrm{e}}}\right)-\left(1-\frac{m_{\mathrm{i}}}{m_{\mathrm{hi}}}\right) \frac{n_{\mathrm{hi} 0}}{n_{0}}}+\right.} \\
& \left.\left(\begin{array}{l}
1-\frac{n_{\mathrm{hi} 0}}{n_{0}} \\
\left(1+\frac{m_{\mathrm{i}}}{m_{\mathrm{e}}}\right)-\left(1-\frac{m_{\mathrm{i}}}{m_{\mathrm{hi}}}\right) \frac{n_{\mathrm{hi} 0}}{n_{0}}
\end{array}\right]^{1 / 2} \frac{T_{\mathrm{i}}}{T_{\mathrm{e}}}\right]^{1 /} .
\end{aligned}
$$

We consider the values of plasma parameters in the ionospheres as: $m_{\mathrm{i}} / m_{\mathrm{e}}=2000, m_{\mathrm{i}} / m_{\mathrm{hi}}=1 / 16, \gamma=3, T_{\mathrm{e}}=2 \mathrm{eV}$, $m_{\mathrm{i}}=m_{\mathrm{p}}=1.6726 \times 10^{-27} \mathrm{~kg}$. Then we find $C_{\mathrm{s}}=1.38 \times 10^{4}$ $\mathrm{m} \cdot \mathrm{s}^{-1}$ and due to $m_{\mathrm{i}} / m_{\mathrm{e}}$ is large enough, the formula (17) can be simplified as following:

$$
\begin{aligned}
\lambda=\lambda\left(n_{0}, n_{\mathrm{hi} 0}, T_{\mathrm{i}}, T_{\mathrm{e}}\right)=\left[\frac{m_{\mathrm{i}}}{m_{\mathrm{e}}} \frac{1-\frac{n_{\mathrm{hi} 0}}{n_{0}}}{\left.1+\frac{m_{\mathrm{i}}}{m_{\mathrm{e}}}\right)-\left(1-\frac{m_{\mathrm{i}}}{m_{\mathrm{hi}}}\right) \frac{n_{\mathrm{hi} 0}}{n_{0}}}+\right. \\
{\left.\left[\begin{array}{l}
\left.\left.1-\frac{1-\frac{n_{\mathrm{hi} 0}}{n_{0}}}{\left(1+\frac{m_{\mathrm{i}}}{m_{\mathrm{e}}}\right)-\left(1-\frac{m_{\mathrm{i}}}{m_{\mathrm{hi}}}\right) \frac{n_{\mathrm{hi} 0}}{n_{0}}}\right) \gamma \frac{T_{\mathrm{i}}}{T_{\mathrm{e}}}\right]^{1 / 2} \approx \\
1-\frac{n_{\mathrm{hi} 0}}{n_{0}}+\left(1-\frac{n_{\mathrm{hi} 0}}{n_{\mathrm{hi} 0}}\right. \\
\left.1+\frac{m_{\mathrm{i}}}{n_{0}} \cdot \frac{1-n_{\mathrm{hi} 0} / n_{0}}{1+n_{\mathrm{hi} 0} / n_{0}}\right)
\end{array}\right]^{\frac{T_{\mathrm{i}}}{m_{\mathrm{e}}}}\right]^{1 / 2} \approx }
\end{aligned}
$$

$$
\left[\frac{1-\frac{n_{\mathrm{hi} 0}}{n_{0}}}{1+\frac{n_{\mathrm{hi} 0}}{n_{0}}}+\gamma \frac{T_{\mathrm{i}}}{T_{\mathrm{e}}}\right]^{1 / 2} .
$$

In the observations of DEMETER $T_{\mathrm{i}} \approx T_{\mathrm{e}}$. Figure 1 shows the decrease of the ion acoustic speed with increase of heavy ion density.

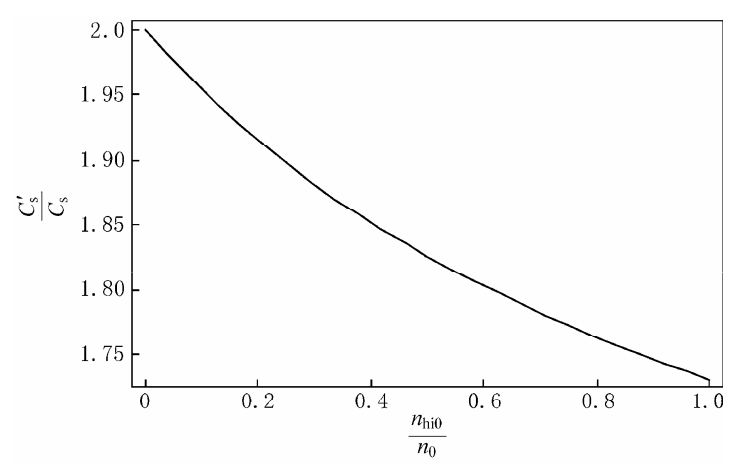

Figure 1 Ion acoustic speed $C_{\mathrm{s}}^{\prime} / C_{\mathrm{s}}$ as a function of heavy ions $n_{\mathrm{hio}} / n_{0}$.

\section{Nonlinear dynamics of ion-acoustic solitons}

For the arbitrary amplitude of density perturbations we study the nonlinear dynamics. It is considered that the earthquake emission produces the density perturbations that creates an electrostatic field $\boldsymbol{E}=-\nabla \phi$, where $\phi$ is the electrostatic potential. The electrons and the heavy ions obey the Boltzmann distribution:

$$
n_{\mathrm{e}}=n_{\mathrm{e} 0} \exp \left(\frac{e \phi}{T_{\mathrm{e}}}\right)
$$

and

$$
n_{\mathrm{hi}}=n_{\mathrm{hi} 0} \exp \left(\frac{-e \phi}{T_{\mathrm{hi}}}\right),
$$

where, $T_{\mathrm{e}}$ and $T_{\mathrm{hi}}$ are the electron's and heavy ion's temperatures, respectively.

The approach has been used to get Boltzmann distributions for the case of electron:

$$
n_{\mathrm{e}} m_{\mathrm{e}}\left[\frac{\partial \boldsymbol{u}_{\mathrm{e}}}{\partial t}+\boldsymbol{u}_{\mathrm{e}} \cdot \nabla \boldsymbol{u}_{\mathrm{e}}\right] \approx n_{\mathrm{e}} m_{\mathrm{e}} \frac{\partial \boldsymbol{u}_{\mathrm{e}}}{\partial t} \approx-e n_{\mathrm{e}} \boldsymbol{E}-\nabla p .
$$

For $m_{\mathrm{e}} \rightarrow 0$,

$$
e \nabla \phi=\frac{\nabla p}{n_{\mathrm{e}}}=\frac{\gamma T_{\mathrm{e}}}{n_{\mathrm{e}}} \nabla n_{\mathrm{e}}=\gamma T_{\mathrm{e}} \cdot \nabla \ln n_{\mathrm{e}},
$$




$$
\ln n_{\mathrm{e}}=\frac{e \phi}{\gamma T_{\mathrm{e}}}+c
$$

and

$$
n_{\mathrm{e}}=n_{\mathrm{e} 0} \exp \left(\frac{e \phi}{\gamma T_{\mathrm{e}}}\right)=n_{\mathrm{e} 0} \exp \left(\frac{e \phi}{T_{\mathrm{e}}}\right)
$$

where $n_{\mathrm{e} 0}=\exp c$ and $\gamma=1$.

However, for $m_{\mathrm{hi}}$ that is much larger than $m_{\mathrm{e}}$, this approach would not be suitable to get the same expression as mentioned above. But we consider the slow motion of heavy ions $\left(\boldsymbol{u}_{\mathrm{hi}} \rightarrow 0\right)$ for what it may be acceptable. tions:

Ion dynamics is governed by the following equa-

$$
\begin{gathered}
\frac{\partial n_{\mathrm{i}}}{\partial t}+\nabla \cdot\left(n_{\mathrm{i}} \boldsymbol{V}_{\mathrm{i}}\right)=0, \\
\frac{\partial \boldsymbol{V}_{\mathrm{i}}}{\partial t}+\left(\boldsymbol{V}_{\mathrm{i}} \cdot \nabla\right) \boldsymbol{V}_{\mathrm{i}}=-\frac{e}{m_{\mathrm{i}}} \nabla \boldsymbol{\phi}
\end{gathered}
$$

and

$$
\nabla^{2} \phi=4 \pi e\left(n_{\mathrm{e}}-n_{\mathrm{i}}-n_{\mathrm{hi}}\right) .
$$

We introduce the following dimensionless variables:

$$
\begin{gathered}
\bar{n}_{\mathrm{i}}=\frac{n_{\mathrm{i}}}{n_{0}}, \quad \bar{n}_{\mathrm{hi} 0}=\frac{n_{\mathrm{hi} 0}}{n_{0}}, \quad \boldsymbol{u}_{i}=\frac{\boldsymbol{V}}{C_{\mathrm{s}}}, \quad \bar{\phi}=\frac{e \phi}{T_{\mathrm{e}}}, \\
\tau=\omega_{\mathrm{p}} t, \quad \bar{x}=\frac{x}{\lambda_{\mathrm{D}}}, \quad \alpha=\frac{T_{\mathrm{e}}}{T_{\mathrm{hi}}},
\end{gathered}
$$

where $\omega_{\mathrm{p}}=\left(4 \pi e^{2} n_{0} / m_{\mathrm{i}}\right)^{1 / 2}$ and $\lambda_{\mathrm{D}}=\left(T_{\mathrm{e}} / 4 \pi e^{2} n_{0}\right)^{1 / 2}$. Considering the one dimensional perturbations $(\nabla \rightarrow \partial / \partial \bar{x})$, the normalized governing equations of ion dynamics are given by

$$
\begin{aligned}
& \frac{\partial \bar{n}_{\mathrm{i}}}{\partial \tau}+\frac{\partial}{\partial \bar{x}}\left(\bar{n}_{\mathrm{i}} u_{\mathrm{i}}\right)=0 \\
& \frac{\partial u_{\mathrm{i}}}{\partial \tau}+u_{i} \frac{\partial u_{\mathrm{i}}}{\partial \bar{x}}=-\frac{\partial \bar{\phi}}{\partial \bar{x}}
\end{aligned}
$$

and

$$
\frac{\partial^{2} \bar{\phi}}{\partial \bar{x}^{2}}=\exp \bar{\phi}-\bar{n}_{\mathrm{i}}-\bar{n}_{\mathrm{hi} 0} \exp (-\alpha \bar{\phi})
$$

To study the properties of stationary arbitrary amplitude of electrostatic waves, we transform the above set of equations to a stationary frame moving with velocity $V$, the phase velocity of the wave, i.e., $\xi=\bar{x}-M \tau$, where $M=V / C_{\mathrm{i}}$ is the Mach number with respect to the ion thermal velocity $C_{\mathrm{i}}=\left(T_{\mathrm{i}} / V_{\mathrm{i}}\right)^{1 / 2}$. Then with the imposed appropriate boundary conditions for the localized disturbances, viz, $u \rightarrow 0, \bar{n}_{i} \rightarrow 1-\bar{n}_{\text {hi } 0}$ at $\xi \rightarrow \pm \infty$ (i.e., away from the centre of localization), from equations (25) and (26), we obtain

$$
\bar{n}_{i}=\frac{\left(1-\bar{n}_{\mathrm{hi} 0}\right) M}{\sqrt{M^{2}-2 \bar{\phi}}} .
$$

Substituting above equation into equation (27) and multiplying both sides of the resulting equation by $\partial \bar{\phi} / \partial \xi$, integrating once, and taking into account the appropriate boundary conditions, i.e., $\bar{\phi} \rightarrow 0$ and $\partial \bar{\phi} / \partial \xi \rightarrow 0$ at $\xi \rightarrow \pm \infty$, we obtain the energy integral

$$
\frac{1}{2}\left(\frac{\partial \bar{\phi}}{\partial \xi}\right)^{2}+\Psi(\bar{\phi}, M)=0
$$

where $\Psi(\bar{\phi}, M)$ is the Sagdeev potential, that is,

$$
\begin{gathered}
\Psi(\bar{\phi}, M)=1+\left(1-\bar{n}_{\mathrm{hi} 0}\right) M^{2}-\exp \bar{\phi}- \\
\left(1-\bar{n}_{\mathrm{hi} 0}\right) M \sqrt{M^{2}-2 \bar{\phi}}+\frac{\bar{n}_{\mathrm{hi} 0}}{\alpha}[1-\exp (-\alpha \bar{\phi})] .
\end{gathered}
$$

The solitonic solution of equation (29) exists when the usual conditions, namely, $\Psi(\bar{\phi}, M)=0$ and $\partial \Psi(\bar{\phi}, M) / \partial \bar{\phi}=0$ at $\bar{\phi}=0$, and $\Psi(\bar{\phi}, M)<0$ for $0<|\bar{\phi}|<\left|\bar{\phi}_{0}\right|$, where $\left|\bar{\phi}_{0}\right|$ is the maximum amplitude of the soliton, are satisfied. Equation (28) defines that $\left|\bar{\phi}_{0}\right|=M^{2} / 2$. The condition $\partial^{2} \Psi(\bar{\phi}, M) / \partial \phi^{2}<0$ at $\bar{\phi}=0$ is satisfied provided $M>M_{0}$, where $M_{0}=$ $\left(1-\bar{n}_{\text {hi } 0}\right)^{1 / 2}$ is the lower critical Mach number. For $\bar{n}_{\text {hi } 0}=0.01$, we have $M_{0}=0.99$.

The upper critical Mach number can be determined from the conditions that at $\bar{\phi}=\bar{\phi}_{0}=M^{2} / 2, \partial \bar{\phi} / \partial \xi=0$ (i.e., at the maximum amplitude of $\bar{\phi}$ the derivative is zero). Then from equation (29) $\Psi\left(\bar{\phi}_{0}, M\right)=0$, which yields the following equation for $M$ :

$$
\begin{aligned}
& \exp \left(\frac{M^{2}}{2}\right)-1-\left(1-\frac{\bar{n}_{\mathrm{hi} 0}}{2}\right) M^{2}- \\
& \frac{\bar{n}_{\mathrm{hi} 0}}{\alpha}\left[1-\exp \left(-\alpha \frac{M^{2}}{2}\right)\right]=0 .
\end{aligned}
$$

Numerically we solve the equation (31) for particular values of $\bar{n}_{\text {hi } 0}=0.01$ and $\alpha=0.1$. We find the 
upper Mach number $M=M_{\max }=1.6$. Thus it seems that ion acoustic soliton may be developed in the perturbed ionospheric plasma with the Mach number between $0.99<M<1.6$. Figure 2 shows the Sagdeev potential $\Psi(\bar{\phi}, M)$ for $\alpha=0.1, \bar{n}_{\mathrm{hi} 0}=0.01$ and $M=1.5$.

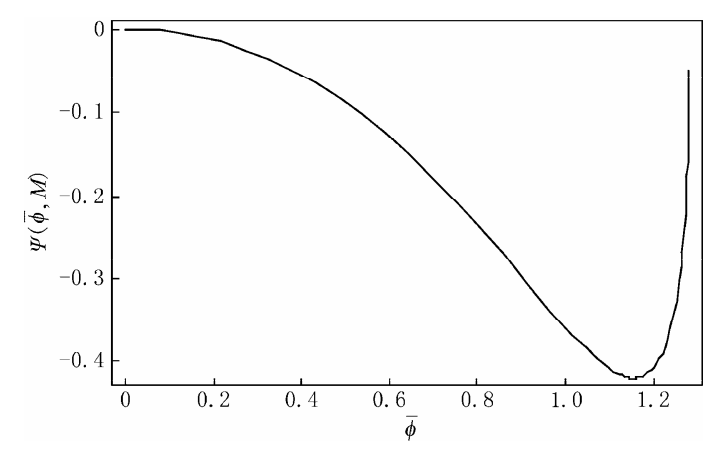

Figure 2 Sagdeev potential $\Psi(\bar{\phi}, M)$ for $\bar{n}_{\mathrm{hi} 0}=0.01$, $\alpha=0.1$ and $M=1.5$.

To confirm the above prediction, we numerically solved the equation

$$
\frac{\partial^{2} \bar{\phi}}{\partial \xi^{2}}=\exp \bar{\phi}-\frac{\left(1-\bar{n}_{\mathrm{hi0}}\right) M}{\sqrt{M^{2}-2 \bar{\phi}}}-\bar{n}_{\mathrm{hi} 0} \exp (-\alpha \bar{\phi})
$$

with the boundary conditions:

$$
\left.\frac{\partial \bar{\phi}}{\partial \xi}\right|_{\xi=0}=0
$$

and

$$
\left.\bar{\phi}\right|_{\xi=0}=\frac{M^{2}}{2} .
$$

Figure 3 shows the numerical solution which is an ion acoustic soliton. It confirms that ion-acoustic solitons may develop in the ionospheric plasma due to earthquake emission.

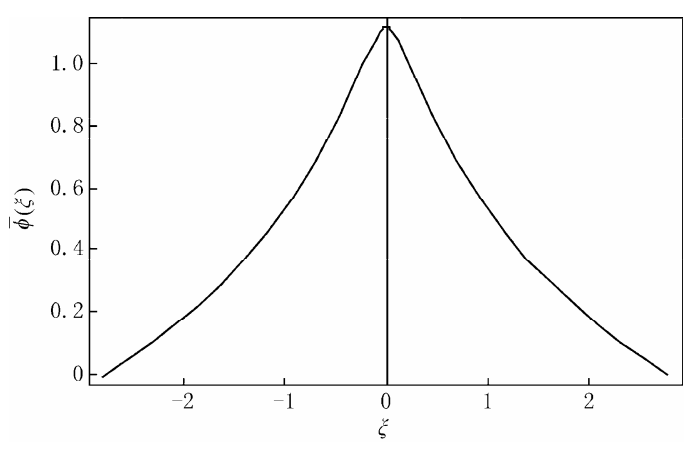

Figure 3 Ion-acoustic soliton of $\bar{\phi}(\xi)$ for $M=1.5$ and $n_{\text {hi } 0}=0.01$.

\section{Discussion and conclusions}

DEMETER satellite launched is already more than four years. The national oceanic and atmospheric administration (NOAA) and the national aeronautics and space administration (NASA) jointly developed a series of polar operational environmental satellites (POES). A decade of NOAA-15 particle flux data is now available. Presently, it offers an opportunity to test the correlations between seismic activity and effects on the ionosphere. In this study, we try to find the correlation in detecting the soliton like structure rather than the particle flux. For this we took the available data collected on DEMETER satellite during the Pu'er earthquake occurred in Pu'er region, Yunnan province, China in 2007. The data shows that the ionospheric perturbations occurred in a three-component plasma consists of ions, electrons and heavy ions. We consider a low temperature, un-magnetized and collisionless plasma perturbations due to earthquake emission. Since electrons are highly oscillating, so ion dynamics is more predominant in the slow plasma response. Therefore, in this study we concentrate on ion acoustic waves, which are related with the compression of plasma fluids. The dispersion relation for the ion acoustic wave is derived, which shows that the presence of heavy ions decreases the phase velocity of the wave. For arbitrary amplitude of the perturbations, we investigate the nonlinear dynamics. Sagdeev potential is calculated and it is found that soliton like structure may develop within a range of Mach number determined by the presence of heavy ions. The corresponding equation is solved numerically for a particular value of the Mach number and the heavy ions. The solution is an ion-acoustic soliton, which may be detected as a precursor of earthquake prediction. Thus, our earlier study (Mofiz, 2006) on soliton formation in the ionosphere by earthquake emission is further confirmed through this investigation.

There has been published many papers about similar anomalies (Parrot, 1995; Sarkar et al, 2007; Ouyang et al, 2008). But the data of Pu'er earthquake (Zhu et al, 2008) is found easier to develop the multi-component plasma fluid concept in the ionosphere. The electric field wave, electron, ion $\left(\mathrm{H}^{+}\right)$and heavy ion $\left(\mathrm{O}^{+}\right)$density and temperature have been detected on DEMETER. These data will be utilized for a real analysis on soliton formation in our further investigations. It is to be noted that there may be other factors that can result in the variation of plasma but the formation of solitary pulses 
may be one of them.

\section{References}

Berthelier J J, Godefroy M, Leblanc F, Malingre M, Menvielle M, Lagoutte D, Brochot J Y, Colin F, Elie F, Legendre C, Zamora P, Benoist D, Chapuis Y, Artru J and Pfaff R (2006). ICE, the electric field experiment on DEMETER. Planet Space Sci 54: 456-471.

Mofiz U A (2006). On the generation of large amplitude spiky solitons by ultralow frequency earthquake emission in the Van Allen radiation belt. Physics of Plasmas 13: 082902(1-10).

Parrot M (1995). Use of satellites to detect seismo-electromagnetic effects. $A d v$ Space Science Res 15(11): 27.

Parrot M, Benoist D, Berthelier J J, Blecki J, Chapuis Y, Colin F, Elie F, Fer- geau P, Lagoutte D, Lefeuvre F, Legendre C, Lévêque M, Pincon J L, Poirier B, Seran H-C and Zamora P (2006). The magnetic field experiment IMSC and its data processing on board DEMETER: Scientific objectives, description and first results. Planet Space Sci 54: 441-455.

Sarkar S, Gwal A K and Parrot M (2007). Ionospheric variations observed by the DEMETER satellite in the mid-latitude region during strong earthquakes. J Atmos Sol-Terr Phys 69(13): 1 524-1 540.

Ouyang X Y, Zhang X M, Shen X H, Liu J, Qian J D, Cai J A and Zhao S F (2008). Ionospheric $N_{\mathrm{e}}$ disturbances before 2007 Pu'er, Yunnan, China, earthquake. Acta Seismologica Sinica 21(4): 425-437.

Zhu R, Yang D M, Jing F, Yang J Y and Ouyang X Y (2008). Ionospheric perturbations before Pu'er earthquake observed on DEMETER. Acta Seismologica Sinica 21(1): 77-81. 